\title{
Gene and Stem Cell Therapy in Erectile Dysfunction
}

\author{
Trevor Hardigan, R. Clinton Webb and Kenia Pedrosa Nunes \\ Georgia Health Sciences University, Augusta, Georgia \\ USA
}

\section{Introduction}

Erectile dysfunction (ED) is a complex, multifactorial issue commonly affecting men of all ages. ED is currently defined as "the inability to achieve and/or maintain penile erection sufficient to permit satisfactory sexual intercourse", and has been associated with such risk factors as hypertension, diabetes, alcoholism, smoking, and pelvic surgery [1]. This condition has seriously impacted the men's quality of life. Even though nowadays there are a large number of options to treat ED, a considerable number of patients do not answer adequately the conventional therapies available. Penile erection is achieved through a neurovascular response exhibiting an increase in arterial inflow, relaxation of corporal smooth muscle, and restriction of the venous outflow. Relatively recent pharmacological advances, namely oral phosphodiesterase-5 (PDE5) inhibitors, have become the first line of treatment in ED. PDE5 inhibitors allow the patient to achieve a penile erection via the effects on the nitric oxide (NO) signaling pathway, which is the principal mediator of corporal smooth muscle relaxation. The inhibition of the degradative actions of PDE5 on 3',5'-cyclic guanosine monophosphate (cGMP), the second messenger molecule of NO signaling, lead to an increased bioavailability of $\mathrm{NO}$ in the corporal smooth muscle thus promoting vasodilation and penile erection. However, despite the widespread efficacy of these medications, there are still men for which oral PDE5 inhibitors are ineffective. ED in men with diabetes, for example, is typically more severe, exhibiting a less effective response to PDE5 inhibitors when compared to non-diabetic patients. The vascular injuries that accompanies diabetes is often too damaging to endothelial physiology to allow adequate penile erections [4] and thus complicates use of the oral medications as treatment for ED. Due to the vast array of potential causes of ED ranging from neurogenic and vasculogenic to hormonal issues, there exists a continuing need to address these deficiencies in patient management of ED and pursue new treatments. Two avenues showing a great deal of promise in ED treatment are gene therapy and stem cell therapy, both of which could possibly provide methods to prevent or even cure ED. This chapter will focus on recent studies in both gene and stem cell therapies in ED research, with the objective to establish that continued scientific exploration into these fields will yield clinically applicable approaches to combat this condition.

\section{Gene therapy and ED}

Gene therapy is the introduction of exogenous genetic material into cells that either restores or enhances normal cellular function that is defective, or essentially attenuates the functional 
effects of the mutant genetic phenotypic expression. This is accomplished through the use of vectors, which are designed to deliver or transfect a given gene into an organism allowing the organism to subsequently reproduce the gene and its protein product. Many different types of vectors have been characterized; viral vectors such as retroviruses and adenoviruses, cell-based vectors such as myoblasts and endothelial cells, as well as non-viral vectors such as plasmid DNA and naked DNA. Viral vectors are commonly used due to their high cellular transfection efficiency; however they have been shown to trigger immune and inflammatory responses which can reduce their effects [5]. The host's immune response can generate antibodies to the virus being used as vector, prompting destruction of the delivery system before it generates a therapeutic effect [1]. Additionally, many of the viruses used as vectors do not exhibit tissue specificity, contributing to issues associated with viral migration through the systemic circulation to other unwanted tissues or organ systems [1].

Several molecules have been examined as potential gene therapeutic approaches for ED, including vascular endothelial growth factor (VEGF), NO synthase, precalcitonin generelated peptide, brain derived neurotrophic factor, and the calcium sensitive potassium (maxi-K) channel [5]. The penis is considered to be a well suited structure for gene therapy due to its limited blood supply, which would decrease the risks associated with non-target infection via the systemic circulation. Its external positioning allows for an ease of accessibility for genetic manipulation, and the intracellular transference of the erectile tissue response to the delivery by gap junctions of corporal smooth muscle further make gene therapy a useful approach as only a proportion of cells are required to be transfected [5]. The relatively low turnover rate of vascular smooth muscle cells indicates the potential for long term expression of introduced genes and thus would yield an improvement over the short term effects of current pharmacological therapies [4]. The role of NO in regulating corporal smooth muscle relaxation is a primary area of concern in ED, and consequently a great deal of the research examining potential gene therapeutic treatments focuses on ways to repair deficient endothelial NO production. [4]

\section{1 eNOS gene therapy}

eNOS, or nitric oxide synthase 3, is an enzyme found in endothelial cells that produces the $\mathrm{NO}$ required for relaxation of the surrounding smooth muscle. It has been shown in patients with ED that a lack of eNOS expression potentiates the difficulties in achieving and maintaining adequate penile erection through low bioavailability of $\mathrm{NO}$ and subsequent increase in vascular tone [4]. There have been several studies examining the role of eNOS gene therapy to treat many of the underlying vascular pathologies leading to ED. Champion et al. studied the effects of using adenoviral gene transfer of eNOS in an age-related rat model of ED. Through the increase in production of eNOS mRNA and subsequent increase in protein expression, it was observed that there was a notable increased erectile response to cavernous nerve stimulation, PDE5 inibitors, and also acetylcholine post-treatment. Within the aged-penis there was also an increase in the presence of cGMP noted. In diabetic rat models, intracavernosal pressure recorded during cavernosal nerve stimulation is markedly lower when compared to the control animals [1]. Bivalacqua et al. have shown that the use of intracavernous eNOS transfection is also a viable therapy, improving both the erectile response to cavernous nerve stimulation and the levels of corporal NO in diabetic rat model of ED. These findings were assessed in conjunction with the use of a PDE5 inhibitor in the 
diabetic rat model, and it was observed that when combined with intracavernous eNOS transfection the erectile response to the pharmacologic treatment was increased along with levels of cGMP in the penis.

\subsection{VEGF gene therapy}

Vascular endothelial growth factor (VEGF) is a multifunctional protein and a critical mediator of endothelial and smooth muscle physiology. It stimulates angiogenesis and increases vascular permeability as well inhibits apoptosis, and as such it provides a potential therapeutic approach for the treatment of ED by increasing NO levels through the expansion of penile vasculature [1]. The increase in vasculature would bring about an accompanying increase in endothelial cells, thus creating an increase of NO production in the target organ. Both VEGF and its receptor flk-1 have been shown to be downregulated in certain types of ED [4], suggesting a possible pathological association. There have been several studies indicating the potential benefits of VEGF treatment for ED. Intracavernous injection of VEGF has been shown to provide a protective effect against hypercholesteremia in penile corporal endothelium from an atherosclerotic rabbit model of ED. This protective effect of VEGF has also been shown in other models of ED such as those accompanying diabetes or in traumatic arteriogenic ED. Additionally, endothelial hypertrophy and hyperplasia were observed following intracavernous VEGF protein delivery in another hypercholesteremic ED model [4]. It is believed that the anti-apoptotic effects of VEGF are associated with this hyperplasia and hypertrophy of endothelial cells, potentially by directly inducing anti-apoptotic pathways in the endothelium. Other potentially therapeutic effects of VEGF in connection with ED are an increase in the expression of eNOS and a stimulatory effect on eNOS phosphorylation.

Further studies have utilized the data supporting the benefits of VEGF protein introduction as a foundation for designing VEGF gene therapy treatments. The goal was that introducing VEGF DNA would increase long-term expression of VEGF and ameliorate any deficiencies in protein amount, essentially acting as a curative treatment for ED [4]. In a study by Rogers et al., an adeno-associated virus was used to transfect VEGF DNA into a model of venogenic $\mathrm{ED}$, with resulting active angiogenesis indicated by endothelial cell hypertrophy and hyperplasia. A similar study examining VEGF DNA delivery in a venogenic model of ED using an adenovirus was shown to increase phosphorylation of eNOS leading to a concurrent increase in the bioavailability of $\mathrm{NO}$ and recovery of erectile function [10]. VEGF introduction has also been examined using a combined gene therapy approach with angiopoetin-1, an angiogenic growth factor that promotes the creation of new blood vessels from pre-existing vessels. Using an adenovirus for both VEGF and angiopoetin-1 in a hypercholesteremic rat model of ED, it was found that the ratio of phosphorylated eNOS to total eNOS was markedly higher in animals receiving the gene therapy versus the control animals. The combined gene therapy approach was found to increase factor VIII-positive endothelial density with a subsequent increase in erectile response to electrical stimulation, suggesting the importance of functional endothelium in management of ED.

\subsection{SOD gene therapy}

Another potential target for gene therapy in ED is utilizing superoxide dismutase (SOD) to decrease the levels of reactive oxygen species (ROS). Reactive oxygen species have been 
shown to play a critical role in endothelial cell dysfunction, primarily through the actions of superoxide scavenging of $\mathrm{NO}$ to form the toxic substance peroxynitrite [4]. Superoxide and peroxynitrite create a cascade of ROS formation by uncoupling eNOS within the endothelium, decreasing the production of $\mathrm{NO}$ and thus impairing erectile function. This creation of ROS is exacerbated by the expression of inducible nitric oxide synthase (iNOS) during oxidative stress conditions, leading to the production of even more ROS. Antioxidants are involved in attenuating the toxic effects of ROS. SOD, found in cells such as vascular endothelium, helps to decrease levels of superoxide by catalyzing the dismutation of the ROS into hydrogen peroxide and water [4].

In a study by Bivalacqua et al., intracavernous adenoviral gene transfer of extracellular SOD was performed in an age-related rat model of ED in an attempt to decrease the levels of ROS. In the rat model there was an observed increase in superoxide production, a decrease in cGMP production as well as a decrease in erectile response to cavernous nerve stimulation. Additionally, they used nitrotyrosine staining as a measure of oxidative stress and found elevated levels in the aged rats. Following transfection of extracellular SOD DNA, there was an increase in extracellular SOD mRNA, protein, and activity levels, along with an increase in cGMP production. Oxidative stress was again measured using nitrotyrosine staining and a decrease was observed post therapeutic treatment. Erectile response to cavernous nerve stimulation was also restored, suggesting that the introduction of extracellular SOD DNA holds potential a viable gene therapy treatment to combat ED resulting from ROS damage to penile endothelium. It has also been shown that ROS play a role in diabetes-related vascular dysfunction and also ED [4], prompting studies on the effects of extracellular SOD gene therapy in diabetic models of ED. Extracellular SOD expression was notably decreased in the diabetic penis, contributing to an increase in the levels of ROS present. Adenoviral transfection of extracellular SOD decreased levels of superoxide and increased cGMP production, which together improved erectile function in an endothelium dependent manner.

\subsection{Anti-arginase gene therapy}

Supplementation of the amino acid L-arginine, either through the diet or direct infusion, has been shown to cause an increase in $\mathrm{NO}$ and a subsequent enhancement of endotheliumrelated vasodilation in the penis [2]. L-Arginine is used as a substrate by both eNOS and arginase, the latter of which converts L-arginine to urea and L-ornithine. This creates a synergistic relationship between the two enzymes, as an increase in arginase activity leads to a downregulation of NO biosynthesis through competition with eNOS [2]. The enzyme arginase has two isoforms, arginase I and arginase II. Arginase I and II are commonly referred to as the hepatic type and extrahepatic type, respectively, though in a study by Bivalacqua et al. which showed an increase in arginase activity in human diabetic corpus cavernosum that both arginase isoforms were found. It was suggested that the impaired endothelium-derived NO bioactivity or signaling via an increased expression of arginase plays a role in the reduction of endothelium-dependant response in the penis of aged mice [2]. Utilizing adeno-associated viral gene transfer of anti-arginase, Bivalacqua et al. found that there was a decrease of arginase- 1 protein and mRNA in the aged mouse penis accompanied by restoration of endothelial and erectile function in vivo [2]. The increase in erectile function was attributed to the increase in constitutive NOS activity and penile cGMP 
levels, suggesting that arginase can interfere with normal erectile function by decreasing production of endothelium-derived eNOS in the penis. Interestingly, it has been shown that in the aged rat penis there is an increase in eNOS protein expression in endothelial cells due to a reduction in caveolin-1, a protein that regulates eNOS. This increase in eNOS protein expression is accompanied by a significant decrease in eNOS activity, suggesting that the upregulated protein is not biologically active. This is supported by the restoration of endothelial vascular responses as a result of increased eNOS activity following adenoassociated viral gene transfer of anti-arginase [2]. While arginase inhibition was shown to improve erectile function, it was not shown to fully restore it, suggesting that there are likely multifactorial mechanisms of endothelial dependant erectile response impairment. Even still, the enhanced penile eNOS activity and cGMP levels resulting from arginase inhibition via anti-arginase gene therapy make it a potential molecular therapeutic treatment of ageassociated vasculogenic ED [2].

When considering gene therapy as a treatment for ED, there are a number of potential issues that need to be taken into consideration. As mentioned previously, there is the risk that viral vectors could enter the systemic circulation and thus cause random transgene expression in unwanted tissues or organs. Increasing the specificity of viral vectors, such that they could target appropriate cells without damaging other cells, could allow for injection systemically rather than intracavernously. There are several methods that have been examined by which to increase the specificity of the viral vectors such as transcriptional and transductional targeting. Transcriptional targeting works through the selection of a cell specific promoter, whereas transductional targeting utilizes cell specific membrane markers to induce delivery of the genetic material. The risk of an immune response to the commonly studied viral vectors is also a concern, as this could hinder use of the therapy in both, acute or repeated clinical treatments. To limit the possibility of an unwanted immune and inflammatory response, it would be beneficial to lower the necessary load of virus required to transduce cells in the penis. Future studies exploring gene therapy for the treatment of ED should look to create as normal of an erectile response as possible by maximizing the specificity of the treatments as well as decreasing any potential unwanted side effects.

\section{Stem cell therapy and ED}

Stem cells have become increasingly popular as a potential treatment for a wide variety of diseases, including ED. Stem cells are defined by the capacity to self renew and differentiate into one or more distinct cell types. The stem cell's potential to differentiate can be extensive, being referred to as pluripotent when it can differentiate into any cell type in the organism. Other levels of differentiation ranging from multipotent to the more limited progenitor cells exist, providing numerous avenues from which potential therapies can be pursued. With the advent of induced pluripotential stem cells (iPS cells), scientists now have access to a line of pluripotent cells with which to work without many of the moral and ethical concerns revolving around the use of human embryonic stem cells. Adult-derived stem cells are also an area of current research, utilizing cell types such as mesenchymal stem cells and adipose-derived stem cells to replace lost or damaged cells in target tissues [2]. Stem cell treatment is attractive as a potential therapy for ED due to the underlying multifactorial pathologies responsible for the disease. The ability to replace whole cells could correct several of the associated specific molecular pathologies of ED with one type of 
treatment [2]. Stem cell replacement of penile endothelial cells may allow for recovery of normal erectile response, as endothelial dysfunction is one of the primary causes of ED.

\subsection{MSC therapy}

One of the more commonly used adult stem cells is mesenchymal stem cells (MSCs). There are produced in the bone marrow, as well as certain other tissues and organs, and they possess the ability to differentiate into a wide variety of cell types depending on the biological environment [2]. They are able to be isolated and expanded ex vivo, making them an advantageous choice for cell therapy [3]. It has been shown previously that MSCs are able to differentiate into endothelial and smooth muscle cells to repair vascular injury in vivo, making them a viable option for the treatment of vasculogenic ED. In a study by Bivalacuqa et al., MSCs were used to differentiate into endothelial cells, thereby improving endothelium-derived NO bioavailability and thus erectile physiology. The MSCs were injected intracavernously with or without simultaneous eNOS gene therapy, and in both groups there was a marked increase in erectile response, eNOS activity, and cGMP levels. The MSCS were found to still be present and expressing endothelial and smooth muscle cell markers not present before injection twenty-one days post injection, which suggests that the differentiated stem cells could provide long term replacement of damaged cells. Other studies have also examined the combined use of MSCs and gene therapy. In a recent study by Qiu et al., the efficacy of MSCs both alone and in combination with VEGF gene therapy for the treatment of ED in a type 1 diabetic rat model was observed. The survival of engrafted stem cells in the target tissue is always a concern during treatments, including in this study following intracavernous implantation. Survival of MSCs has been shown to increase when coupled with VEGF treatment, as noted by the accompanying increase in prosurvival factors phosphorylated Akt and Bcl-xL. Improved erectile function and an increase in the number of endothelial and smooth muscle cells were noted post injection, with the rats receiving the combined stem cell and VEGF gene therapy exhibiting the greatest increase compared to the animals treated with MSCs alone or the control group [3]. This suggests that the use of MSCs is a potential route for stem cell therapy of ED, especially when considered with concurrent gene therapeutic treatments.

\subsection{ADSC therapy}

Another type of adult stem cells that has been examined for the treatment of ED is adiposederived stem cells (ADSCs). They are found in fat tissue and possess the qualities of stem cell, namely self-renewal and differentiation into multiple cell phenotypes, as well as the ability to provide functional repair of damaged tissue. In terms of differentiation and therapeutic potential, ADSCs are similar to MSCs but are easier and safer to harvest in large quantities as adipose tissue is a readily available biological source. ADSCs have been shown to be vascular precursor stem cells, making them an appropriate choice for stem cell therapy of ED [24]. In a study by Bella et al., the effects of ADSCs to repopulate endothelial and smooth muscle cells in erectile tissue following bilateral cavernous nerve crush injury were examined. Using intracavernosal pressure (ICP) as a measure of erectile function, the animals treated with ADSCs were observed to have higher ICP response and thus a better recovery of erectile function when compared with the control animals. The ability of ADSCs to help restore erectile function in this study was suggested to result from their 
differentiation into local penile cell types, as well through the secretion of growth factors that recruited local stem cells to differentiate, promoted IGF-1 mediated local functional cell growth, and exhibited a neurotrophic effect to promote nerve regeneration [26].

In another study using ADSCs, Garcia et al. tested the use of ADSCs for the treatment of ED in obese type 2 diabetic ZDF rats. These rats were shown previously to have an $85 \%$ prevalence of impotence ( $\mathrm{ICP} \leq 60 \mathrm{cmH} 2 \mathrm{O}$ ) among their population, making them an appropriate model to test the efficacy of ADSCs to survive in-vivo and subsequently restore erectile function. Three weeks after ADSCS injection into the rat penis, there was a noted functional improvement in erectile function when compared with untreated animals, as indicated by an improvement in ICP. It was determined that the effect of the treatment may be a result of an increase in the population of endothelial cells and a decrease in intracorporal tissue apoptosis among the treated rats. The survival of the ADSCs was assessed using BrdU labeling, which did not indicate the presence of many injected ADSCs after three weeks post injection. This suggests that the observed therapeutic effects likely result from elaboration of cytokines and growth factors from the ADSCs as opposed to direct differentiation in local cell types. An increase in nNOS (NOS type 1) was also seen, indicating that there may have been a restorative impact on nitrergic neuron axons and ganglia leading to improved erectile function. However, it is also possible that this increase

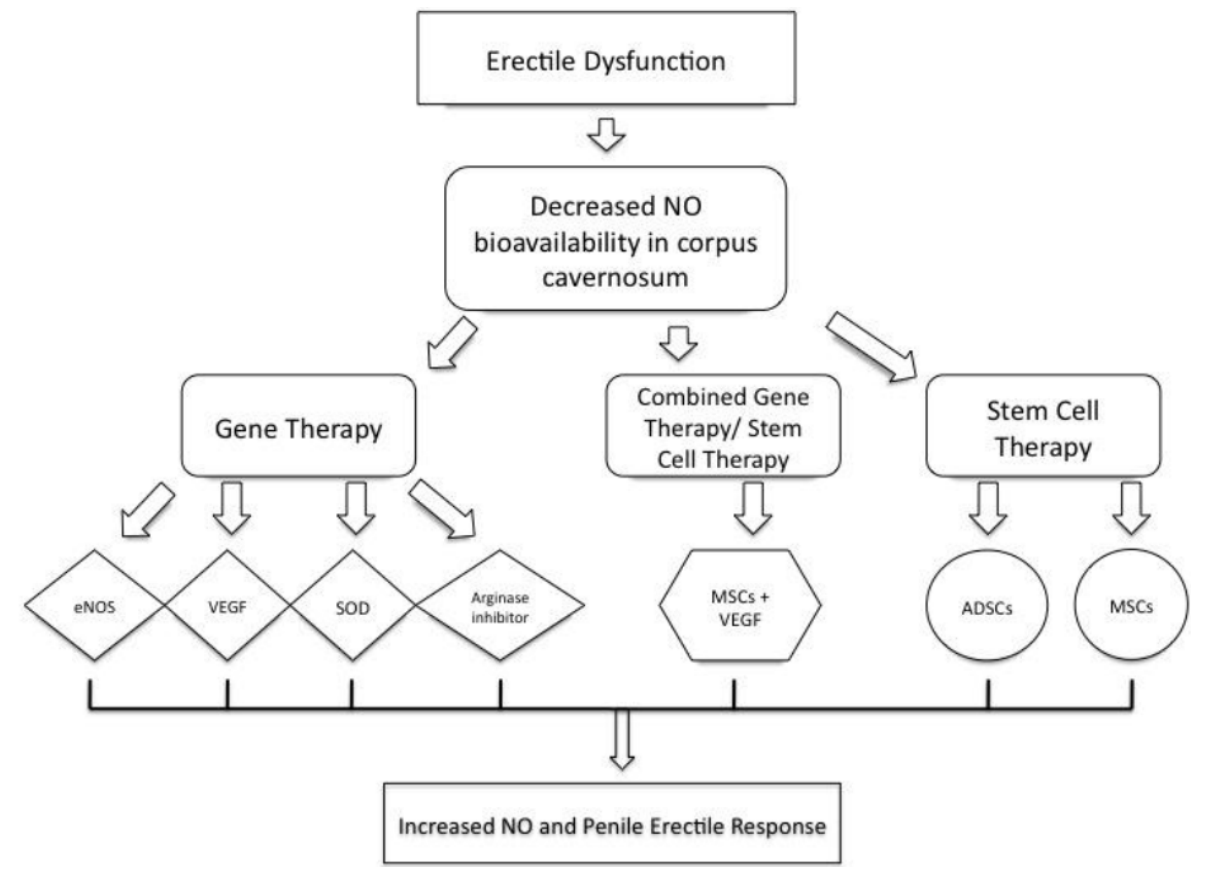

Fig. 1. Gene and stem cell therapies for the treatment of ED. Gene therapy treatments consist of eNOS, VEGF, SOD, and arginase inhibitor vectors. Stem cell therapy treatments include adipocyte-derived stem cell (ADSCs) and mesenchymal stem cell (MSCs) introduction into the cavernosal tissue. A combined gene therapy and stem cell therapy approach utilizing MSCs and VEGF has also been studied. 
in nNOS was a result of increased oxygenated blood flow supplying more dorsal nerve nNOS following erectile function improvement. The use of ADSCs is an attractive therapeutic option in future studies, as the ease with which great numbers of cells can be harvested eliminates the need for ex vivo cell culture, thereby reducing the risk of contamination from a variety of organisms.

\section{Conclusion}

The advent of gene and stem cell therapy has unveiled new horizons for the future of medicine and clinical treatments. Among the many potential diseases able to be positively altered by these therapeutic treatments, ED has already been shown to be an appropriate pathological target. The use of gene and stem cell therapies alone or in combination with each other and other treatments such as pharmacological interventions holds great promise in the future care of patients afflicted with ED (Figure 1).

\section{References}

[1] Mills, J.N., et al., The molecular basis of erectile dysfunction: from bench to bedside. Reviews in urology, 2005. 7(3): p. 128-34.

[2] Bivalacqua, T.J., et al., Overexpression of arginase in the aged mouse penis impairs erectile function and decreases eNOS activity: influence of in vivo gene therapy of anti-arginase. American journal of physiology. Heart and circulatory physiology, 2007. 292(3): p. H1340-51.

[3] Qiu, X., et al., Combined Strategy of Mesenchymal Stem Cells Injection with VEGF Gene Therapy for the Treatment of Diabetes Associated Erectile Dysfunction. Journal of andrology, 2011.

[4] Strong, T.D., et al., Endothelium-specific gene and stem cell-based therapy for erectile dysfunction. Asian journal of andrology, 2008. 10(1): p. 14-22.

[5] Burnett, A.L., Erectile dysfunction management for the future. Journal of andrology, 2009. 30(4): p. 391-6.

[6] Champion, H.C., et al., Gene transfer of endothelial nitric oxide synthase to the lung of the mouse in vivo. Effect on agonist-induced and flow-mediated vascular responses. Circulation research, 1999. 84(12): p. 1422-32.

[7] Bivalacqua, T.J., et al., Gene transfer of endothelial nitric oxide synthase partially restores nitric oxide synthesis and erectile function in streptozotocin diabetic rats. The Journal of urology, 2003. 169(5): p. 1911-7.

[8] Bivalacqua, T.J., et al., Effect of combination endothelial nitric oxide synthase gene therapy and sildenafil on erectile function in diabetic rats. International journal of impotence research, 2004. 16(1): p. 21-9.

[9] Lin, C.S., et al., Intracavernosal injection of vascular endothelial growth factor induces nitric oxide synthase isoforms. BJU international, 2002. 89(9): p. 955-60.

[10] Musicki, B., et al., Phosphorylated endothelial nitric oxide synthase mediates vascular endothelial growth factor-induced penile erection. Biology of reproduction, 2004. 70(2): p. 282-9. 
[11] Rogers, R.S., et al., Intracavernosal vascular endothelial growth factor (VEGF) injection and adeno-associated virus-mediated VEGF gene therapy prevent and reverse venogenic erectile dysfunction in rats. International journal of impotence research, 2003. 15(1): p. 26-37.

[12] Ryu, J.K., et al., Combined angiopoietin-1 and vascular endothelial growth factor gene transfer restores cavernous angiogenesis and erectile function in a rat model of hypercholesterolemia. Molecular therapy : the journal of the American Society of Gene Therapy, 2006. 13(4): p. 705-15.

[13] $\mathrm{Xu}, \mathrm{J}$. , et al., Oxygen-glucose deprivation induces inducible nitric oxide synthase and nitrotyrosine expression in cerebral endothelial cells. Stroke; a journal of cerebral circulation, 2000. 31(7): p. 1744-51.

[14] Bivalacqua, T.J., et al., Gene transfer of extracellular SOD to the penis reduces O2-* and improves erectile function in aged rats. American journal of physiology. Heart and circulatory physiology, 2003. 284(4): p. H1408-21.

[15] Bivalacqua, T.J., et al., Superoxide anion production in the rat penis impairs erectile function in diabetes: influence of in vivo extracellular superoxide dismutase gene therapy. The journal of sexual medicine, 2005. 2(2): p. 187-97; discussion 197-8.

[16] Bivalacqua, T.J., et al., Increased expression of arginase II in human diabetic corpus cavernosum: in diabetic-associated erectile dysfunction. Biochemical and biophysical research communications, 2001. 283(4): p. 923-7.

[17] Bakircioglu, M.E., et al., Decreased trabecular smooth muscle and caveolin-1 expression in the penile tissue of aged rats. The Journal of urology, 2001. 166(2): p. 734-8.

[18] Sadeghi, H. and M.M. Hitt, Transcriptionally targeted adenovirus vectors. Current gene therapy, 2005. 5(4): p. 411-27.

[19] Waehler, R., S.J. Russell, and D.T. Curiel, Engineering targeted viral vectors for gene therapy. Nature reviews. Genetics, 2007. 8(8): p. 573-87.

[20] $\mathrm{Wu}, \mathrm{X}$. , et al., Mesenchymal stem cells participating in ex vivo endothelium repair and its effect on vascular smooth muscle cells growth. International journal of cardiology, 2005. 105(3): p. 274-82.

[21] Wang, T., et al., Cell-to-cell contact induces mesenchymal stem cell to differentiate into cardiomyocyte and smooth muscle cell. International journal of cardiology, 2006. 109(1): p. 74-81.

[22] Bivalacqua, T.J., et al., Mesenchymal stem cells alone or ex vivo gene modified with endothelial nitric oxide synthase reverse age-associated erectile dysfunction. American journal of physiology. Heart and circulatory physiology, 2007. 292(3): p. H1278-90.

[23] Pons, J., et al., VEGF improves survival of mesenchymal stem cells in infarcted hearts. Biochemical and biophysical research communications, 2008. 376(2): p. 419-22.

[24] Lin, G., et al., Potential of adipose-derived stem cells for treatment of erectile dysfunction. The journal of sexual medicine, 2009. 6 Suppl 3: p. 320-7.

[25] Garcia, M.M., et al., Treatment of erectile dysfunction in the obese type 2 diabetic ZDF rat with adipose tissue-derived stem cells. The journal of sexual medicine, 2010. 7(1 Pt 1): p. 89-98. 
[26] Bella, AJ., et al., Non-cell line induced autologous adult adipose tissue derived stem cells enhance recovery of erectile function in the rat following bilateral cavernous nerve crush injury. Sexual Medicine Society of North America Meeting, 2008. 68p. 


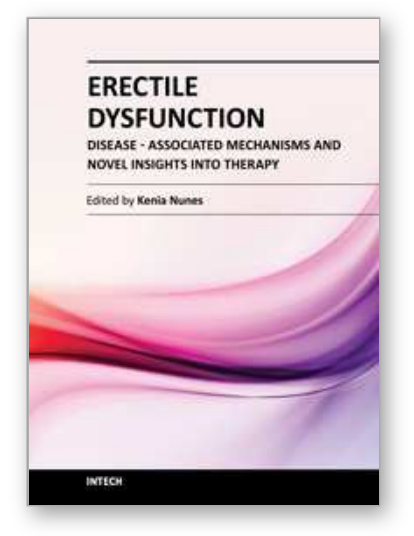

\author{
Erectile Dysfunction - Disease-Associated Mechanisms and Novel \\ Insights into Therapy \\ Edited by Dr. Kenia Nunes
}

ISBN 978-953-51-0199-4

Hard cover, 214 pages

Publisher InTech

Published online 29, February, 2012

Published in print edition February, 2012

Erectile dysfunction is a widespread problem, affecting many men across all age groups and it is more than a serious quality of life problem for sexually active men. This book contains chapters written by widely acknowledged experts, each of which provides a unique synthesis of information on emergent aspects of ED. All chapters take into account not only the new perspectives on ED but also recent extensions of basic knowledge that presage directions for further research. The approach in this book has been to not only describe recent popular aspects of ED, such as basic mechanism updates, etiologic factors and pharmacotherapy, but also disease-associated ED and some future perspectives in this field.

\title{
How to reference
}

In order to correctly reference this scholarly work, feel free to copy and paste the following:

Trevor Hardigan, R. Clinton Webb and Kenia Pedrosa Nunes (2012). Gene and Stem Cell Therapy in Erectile Dysfunction, Erectile Dysfunction - Disease-Associated Mechanisms and Novel Insights into Therapy, Dr. Kenia Nunes (Ed.), ISBN: 978-953-51-0199-4, InTech, Available from:

http://www.intechopen.com/books/erectile-dysfunction-disease-associated-mechanisms-and-novel-insightsinto-therapy/gene-and-stem-cell-therapy-in-erectile-dysfunction

\section{INTECH}

open science | open minds

\section{InTech Europe}

University Campus STeP Ri

Slavka Krautzeka 83/A

51000 Rijeka, Croatia

Phone: +385 (51) 770447

Fax: +385 (51) 686166

www.intechopen.com

\section{InTech China}

Unit 405, Office Block, Hotel Equatorial Shanghai

No.65, Yan An Road (West), Shanghai, 200040, China

中国上海市延安西路65号上海国际贵都大饭店办公楼 405 单元

Phone: $+86-21-62489820$

Fax: +86-21-62489821 
(C) 2012 The Author(s). Licensee IntechOpen. This is an open access article distributed under the terms of the Creative Commons Attribution 3.0 License, which permits unrestricted use, distribution, and reproduction in any medium, provided the original work is properly cited. 